\title{
2020 deutlich weniger Hautkrebs diagnostiziert
}

Die Österreichische Gesellschaft für Dermatologie und Venerologie (ÖGDV) warnte vor Risiken einer späten Erstdiagnose und rief anlässlich des Weltkrebstages am 4. Februar zur Hautkrebsvorsorge auf.

Die Zahl der diagnostizierten Fälle von Hautkrebs war im Jahr 2020 gegenüber 2019 deutlich reduziert. In manchen Monaten wurden bis zu $50 \%$ weniger Basaliome, kutane Plattenepithelkarzinome und Melanome diagnostiziert, wie ein kürzlich im „Journal of the American Academy of Dermatology" erschienener Artikel [1] von Dermatologen aus mehreren amerikanischen Universitätskliniken zeigt.

》) Die Behandlung mit Check-PointInhibitoren muss wegen der COVID-

19-Pandemie nicht verzögert werden

„Dies birgt, insbesondere bei metastasierenden Tumoren wie dem Melanom, das hohe Risiko einer Erstdiagnose in einem bereits fortgeschrittenen Krebsstadium mit einer schlechteren Prognose", erklärt a.o. Prof. Dr.

Quelle: Presseaussendung der ÖGDV

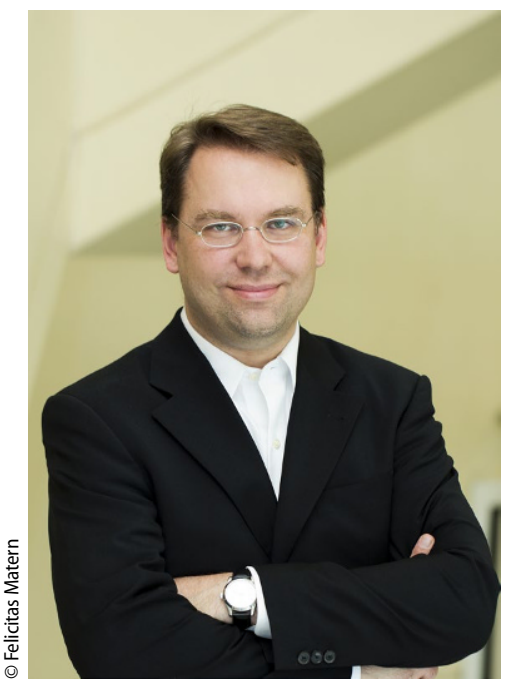

$\Delta$ A. o. Prof. Dr. Christoph Höller
Christoph Höller, Leiter der Arbeitsgruppe Melanom und dermatologische Onkologie der Österreichischen Gesellschaft für Dermatologie und Venerologie.

Anlässlich des Weltkrebstages am 4. Februar appellierte die ÖGDV an die Bevölkerung, die Hautkrebsvorsorge während der Corona-Pandemie keinesfalls zu vernachlässigen. „Bei verdächtigen Hautveränderungen sollte ohne Verzögerung die Hautärztin/der Hautarzt aufgesucht werden, um eine frühzeitige Diagnose und effektive Behandlung von Hauttumoren auch in Zeiten der COVID-19-Pandemie sicherzustellen“, erklärt Höller.

\section{Keine Unterbrechung lebensnot- weniger Therapien}

Die Hauttumorzentren der dermatologischen Abteilungen in Österreich, welche eng im Rahmen der Arbeitsgruppe Melanom und dermatologische Onkologie (AMDO) der ÖGDV kooperieren, konnten trotz aller Einschränkungen durch die Verschiebung von Ressourcen eine Unterbrechung von lebensnotwendigen Therapien vermeiden.

\section{Durch Hautkrebsbehandlung} kein erhöhtes Risiko für schweren COVID-19-Krankheitsverlauf

„Auch bei Patientinnen und $\mathrm{Pa}$ tienten, die aufgrund einer fortgeschrittenen Hautkrebserkrankung eine Immuntherapie mit CheckPoint-Inhibitoren erhalten, besteht kein Grund, die Behandlung wegen der COVID-19-Pandemie zu verzö- gern", sagte Höller. Check-Point-Inhibitoren deaktivieren eine „Bremse“ im Immunsystem, um den Abwehrzellen eine bessere Kontrolle der Tumorerkrankung zu erlauben. Es bestand initial daher die Sorge, dass dies eventuell zu schwereren Verläufen von Patienten mit einer COVID-19-Infektion führen könnte.

In einer internationalen Kooperation [2] konnten nun erstmals die Verläufe von 110 PatientInnen ausgewertet werden, die während einer solchen Therapie eine COVID-19-Infektion hatten. Rund $60 \%$ dieser Patienten wurden wegen eines fortgeschrittenen Melanoms behandelt. Das Ergebnis der Auswertung: Die Rate an symptomatischen Patienten sowie an hospitalisierten Patenten war vergleichbar zur generellen Bevölkerung.

\section{Literatur}

1. Marson JW et al (2021) J Am Acad Dermatol 8:S0190-9622(21)00082-7

2. Rogiers A et al., J Immunother Cancer 9(1):e001931

Hinweis des Verlags. Der Verlag bleibt in Hinblick auf geografische Zuordnungen und Gebietsbezeichnungen in veröffentlichten Karten und Institutsadressen neutral.

\section{hautnah $2021 \cdot 20: 4-5$}

https://doi.org/10.1007/s12326-02100424-y

(c) Springer-Verlag GmbH Austria, ein Teil von Springer Nature 2021 
Hier steht eine Anzeige.

Springer 